\title{
EVOLUCIÓN CIENTÍFICA DE LA ENSEÑANZA DE LENGUAS EN EL CONTEXTO UNIVERSITARIO (1900-2019)
}

\section{SCIENTIFIC EVOLUTION OF LANGUAGE TEACHING IN A UNIVERSITY CONTEXT (1900-2019)}

\author{
Antonio-Manuel Rodríguez-García \\ Universidad de Granada, Espanha \\ arodrigu@ugr.es \\ María Aránzazu Fernández Mora \\ Universidad Nacional de Educación a Distancia (Ceuta), Espanha \\ marfernandez@ceuta.uned.es \\ Antonio José Moreno Guerrero \\ Universidad de Granada, Espanha \\ ajmoreno@ugr.es
}

RESUMEN: Este artículo tiene por objetivo analizar la evolución de la investigación sobre enseñanza de lenguas en el ámbito universitario dentro de la colección principal de la Web of Science (1900-2019). Para ello, se ha desarrollado un estudio bibliométrico por revisión sistemática, tomando en consideración los siguientes indicadores: producción diacrónica, geográfica, autores y de fuente. A su vez, se ha realizado un análisis del desarrollo estructural de los temas sobre una muestra de 2978 referencias procedentes de seis búsquedas combinadas. Los análisis realizados determinan un patrón de crecimiento exponencial de la literatura científica sobre la enseñanza de idiomas en el ámbito universitario durante la última década (2010-2019); período que contempla el $87,2 \%$ de la producción científica actual. En relación a la evolución de los temas del campo, se observa que las líneas de investigación actual se están centrando mayormente en la introducción de la tecnología para el apoyo de la enseñanza de idiomas en el contexto universitario.

PALABRAS CLAVE: enseñanza de idiomas; universidad; educación superior; revisión sistemática.

ABSTRACT: The aim of this article is to analyse the evolution of research on language teaching at the university level within the main collection of the Web of Science (19002019). To this end, a bibliometric study has been developed through systematic review, taking into consideration the following indicators: diachronic, geographical, authors and source productions. At the same time, an analysis of the structural development of the topics has been carried out on a sample of 2978 references from six combined searches. The analyses carried out determine an exponential growth pattern in the scientific literature on language teaching in universities over the last decade (2010-2019), a period that covers $87.2 \%$ of the current scientific production. In relation to the evolution of topics in the field, it is observed that current lines of research are focusing mainly on the introduction of technology to support language teaching in the university context. 
KEYWORDS: language teaching; university; higher education; systematic review.

\section{Introducción}

La enseñanza de idiomas en el ámbito universitario se ha postulado en todo el mundo como un referente esencial en la nueva concepción globalizada de la formación superior (DURHAM; KIM, 2019). En nuestro contexto, la creación del "Espacio Europeo de Educación Superior" (EEES) ha cambiado la concepción de la universidad europea diseñando nuevos planes de estudios que trabajan principalmente con el desarrollo de competencias, entre las que destaca el conocimiento de una lengua extranjera por parte de los estudiantes como aspecto esencial para insertarse activamente en la sociedad del siglo XXI.

Desde una mirada intercontinental, en Japón, el proyecto "Top Global University Project" (TGUP), que surgió para crear universidades orientadas a nivel mundial con el objetivo de aumentar el papel de los idiomas extranjeros en la educación superior, ha dado lugar incluso a la aparición de formas flexibles y únicas de educación en inglés en dichos centros, los cuales están obteniendo grades resultados (ROSE; MCKINLEY, 2018). En la India se precisa la necesidad de continuar con la educación en inglés (MAHAPATRA; MISHRA, 2019). En China se estudia las prácticas lingüísticas de los académicos multilingües en las publicaciones académicas, a la vez que el gobierno amplía la educación multilingüe en la enseñanza superior (ZHENG; GUO, 2019). Sin embargo, al contrario, ocurre en Inglaterra, donde los estudios se centran, entre otros aspectos, en el deficiente historial británico para el aprendizaje de idiomas por el inglés global, ya que las razones utilitarias para aprender idiomas distintos al inglés se ven minadas (LANVERS, 2017; LO BIANCO, 2014).

En cuanto a las variables que se están tomando en consideración actualmente para el aprendizaje de un idioma, destaca la importancia de la motivación (RISUEÑO; VÁZQUEZ; HIDALGO; DE LA BLANCA, 2016); el papel que juegan las emociones de los estudiantes en los procesos que tienen lugar en la adquisición de un segundo idioma (KING, 2016); así como la aptitud innata en la capacidad para aprender un idioma (MANTOU; NOELS, 2017).

Todas estas investigaciones sobre la enseñanza de la lengua extranjera en el ámbito universitario se han visto aún más diversificadas con la introducción del uso de las nuevas tecnologías en este campo. Los estudios se han ido desarrollando en función de la evolución tecnológica en las aulas. En estos últimos años se han dirigido al desarrollo de la influencia de la traducción automática con fines académicos (GROVES; MUNDT, 2015), a los que se involucra a los estudiantes en los procesos de composición multimodal digital, para avanzar en el dominio del idioma, y en el uso de recursos multimodales y herramientas de colaboración para ampliar el público interesado (HAFNER, 2015), e incluso a aquellos que investigan el uso de juegos de computadora para la mejora de la transferencia del vocabulario aprendido (FRANCIOSI, 2017), o el uso de juegos digitales para promover el aprendizaje de la lengua y la alfabetización de hablantes nativos y no nativos (HUNG; YANG; HWANG; CHU; WANG, 2018). Sin desdeñar aquellos trabajos que se centran en el impacto del aprendizaje con los dispositivos móviles en el rendimiento estudiantil (CROMPTON; BURKE, 2018). 
Cómo se puede observar, la enseñanza de una segunda o tercera lengua en el contexto universitario está adquiriendo cada vez mayor relevancia. A su vez, esta área de investigación presenta inmensidad de facetas que justifican la necesidad de un estudio como el que presentamos a continuación.

\section{Método}

Esta investigación tiene por objetivo analizar la evolución de la investigación sobre enseñanza de lenguas en el ámbito universitario dentro de la colección principal de la Web of Science (Science Citation Index Expanded; Social Sciences Citation Index; Arts and Humanities Citation Index; Conference Proceedings Citation Index; Book Citation Index Science, Social Science \& Humanities -; Emerging Sources Citation Index) (1900-2019), dado que es una de las bases de datos de mayor relevancia internacional y que contiene un gran número de referencias y revistas (GIMÉNEZ-ESPERT;PRADO-GASCÓ, 2019). A su vez, este estudio pretende:

- Cuantificar la producción diacrónica de la investigación sobre enseñanza de lenguas en el ámbito universitario (1900-2019).

- Analizar la producción geográfica de la investigación en relación al país e institución que firma el documento científico.

- Estudiar los autores con mayor producción científica al respecto.

- Examinar la productividad de fuente en relación al tipo de publicación, título que la contiene, idioma, área de investigación y obras más citadas.

- Establecer la continuidad de palabras clave entre los periodos establecidos.

- Determinar la evolución científica sobre la enseñanza de lenguas en el ámbito universitario.

- Identificar los temas más relevantes en los tres periodos establecidos para el análisis de la evolución científica (1944-1999, 2010-2014, 2015-2019).

Para la consecución de estos objetivos, se ha seguido una metodología de tipo bibliométrico por revisión sistemática (HINOJO; AZNAR; ROMERO, 2018; TODESCHINI; BACCINI, 2016; RODRÍGUEZ; TRUJILLO; SÁNCHEZ, 2019), mediante el uso de técnicas de rastreo analítico (ÁLVAREZ, 2015) y de cuantificación documental (RODRÍGUEZ, RASO; RUIZ, 2019) mediante una evaluación de rendimiento, en la que se analiza la producción científica de los actores científicos; y la creación de mapas de ciencia, valorando la evolución conceptual del objeto de estudio en un marco longitudinal (COBO; LÓPEZ; HERRERA; HERRERA, 2011).

La investigación bibliométrica sirve para determinar el estado actual de la situación de diversas áreas de conocimiento (QUEVEDO; LÓPEZ, 2010) como ocurre con la enseñanza de idiomas en el ámbito universitario o la enseñanza superior, que nos ocupa. El valor de los estudios bibliométricos de los contenidos presentados por las producciones científicas, atendiendo a la importancia de la difusión del conocimiento para el desarrollo de la ciencia, se revela como un método objetivo y verificable (VELASCO; EIROS; PINILLA; SAN ROMÁN, 2012), y un indicio de calidad para las futuras investigaciones (ZYCH; BUELA, 2010; RUIZ; DELGADO; JIMÉNEZ, 2010). Dichos estudios sobre los distintos ámbitos científicos se muestran significativos para las revistas, al igual que para los 
investigadores, puesto que la producción científica, su difusión y el impacto de las publicaciones y de los investigadores que en ellas trabajan se fundamentan en ellos (ELKINS; MAHER; HERBERT; MOSELEY; SHERRINGTON, 2010; FRANCESCHET, 2010), mostrando las tendencias más innovadoras y recientes, y sirviendo de orientación en posteriores estudios de las áreas de investigación (VELASCO et al., 2012; LÓPEZ; VÁZQUEZ; SARASOLA, 2015).

Para la obtención de las unidades de análisis se empleó una ecuación de búsqueda de palabras clave unidas con el operador booleano "AND" en el tema y dentro de la colección principal de la Web of Science (marzo-junio de 2019). Las mismas fueron extraídas de los tesauros de la UNESCO y ERIC seleccionando: "language education" (LE), "language instruction" (LI) y "language teaching" (LT), "university"(U) y "higher education" (HE) siendo todos ellos elementos clave para el estudio de la enseñanza de idiomas en el contexto universitario. De esta manera, se realizaron seis búsquedas combinadas diferenciadas (LEU, LIU, LTU, LEHE, LIHE y LTHE) que dieron como resultado una muestra final de 2978 documentos.

Para la evaluación de rendimiento y de producción científica se desarrolló un estudio descriptivo, mediante el establecimiento de 9 variables, al que se le aplicó una matriz de protocolo PRISMA-P (HUTTO; CATALÁ; MOHER, 2016) y cuyos criterios de inclusión podemos ver en la Tabla 1.

Tabla 1: Indicador, variable y criterio de inclusión (PRISMA-P).

\begin{tabular}{|c|c|c|}
\hline Indicador & Variable & $\begin{array}{c}\text { Criterios de } \\
\text { inclusión }\end{array}$ \\
\hline Producción diacrónica & Año de publicación & $1900-2019$ \\
\hline Producción geográfica & País & $x \geq 20$ \\
\cline { 2 - 3 } & Institución & $x \geq 10$ \\
\hline \multirow{2}{*}{$\begin{array}{c}\text { Productividad de } \\
\text { autores }\end{array}$} & Documentos por autor & $x \geq 5$ \\
\hline \multirow{4}{*}{\begin{tabular}{c} 
Productividad de fuente \\
\cline { 2 - 3 }
\end{tabular}} & Tipo de fuente & $\mathrm{x} \geq 10$ \\
\cline { 2 - 3 } & Título de fuente & $\mathrm{x} \geq 15$ \\
\cline { 2 - 3 } & Área de investigación & $\mathrm{x} \geq 20$ \\
\cline { 2 - 3 } & Impacto de fuente & $\mathrm{x} \geq 10$ \\
\hline & (citas) & $\mathrm{x} \geq 100$ \\
\hline
\end{tabular}

Para el desarrollo estructural y dinámico del concepto de la enseñanza de las lenguas se en el ámbito universitario se ha desarrollado un análisis de co-palabras (HIRSCH, 2005), fundamentado en el índice-h y en el número de citas (COBO; LÓPEZ; HERRERA; HERRERA, 2011), desarrollando un mapa de ciencia y un análisis de rendimiento para identificar y representar los subdominios conceptuales del campo de investigación y su progreso en la temática de estudio. El análisis de co-palabras longitudinal, establecido con el programa SciMAT (COBO; LÓPEZ; HERRERA; HERRERA, 2012) se estructuró en cuatro fases (MONTERO; COBO; GUTIÉRREZ; SEGADO; HERRERA, 2018):

- Detección de temas de investigación: De las 2978 referencias usadas anteriormente, se ha realizado un mapeo para seleccionar aquellas que solamente tienen palabras 
clave, además de evitar la duplicidad de documentación, desechando el resto de documentos, quedándonos en este caso con un total de 2475 referencias, creando con ello una red de co-ocurrencia mediante nodos. La base son las palabras claves localizadas previamente, las cuales se conectan entre sí cuando dos de estas palabras co-aparecen en diversos textos científicos, generando una red normalizada de co-palabras. Mediante el algoritmo de clustering, se han localizado los temas de investigación, mostrándose así las palabras clave fuertemente relacionadas. Se usaron las palabras claves dadas por los autores y las creadas por WoS, según el tipo de documento. Además, se analizó toda la producción científica para comprobar que no hubiera documentos repetidos, y determinados documentos que aparecieron en la búsqueda que no eran propiamente de la temática tratada, haciendo uso del programa IBM SPSS Statistics 20 , el cual, mediante las tablas frecuencias, localiza los documentos repetidos.

- Representación tema investigación: Para ello se ha usado un diagrama estratégico y una red de temas (CALLON; COURTIAL; LAVILLE, 1991), formada por dos dimensiones (centralidad y densidad), en el que las palabras clave se muestran en cuatro sectores: sector superior derecho, donde alberga los temas motores y fundamentales en el tema de investigación; sector superior izquierdo, alberga las conexiones son débiles y son nodos con poca relevancia en la temática; sector inferior izquierdo, aguarda los temas son relevantes pero no tienen un desarrollo acuciado; y sector inferior derecho, recopila los nodos carecen de desarrollo o relevancia, aunque los que aparecen en dicha zona pueden ser temas emergentes.

- Localización de temas. Esto se determina por la evolución cronológica mostrada por los nodos de un periodo a otro. La fuerza de relación se fundamenta según el número de palabras clave que tienen en común. El análisis se inicia en 1944, dado que el único documento que aparece en las fechas comprendidas entre 1900 y 1909 no recoge palabras clave. Los periodos establecidos han sido 1944-2009, 2010-2014 y 2015-2019.

- Análisis de rendimiento. Cada una de las palabras clave, tienen a su vez, una cadena de conexiones que marcan la tendencia de dicho nodo, ofreciendo datos sobre el uso que hace la comunidad científica sobre la misma, para ello se estableció unos protocolos de análisis, establecidos en la Tabla 2.

Tabla 2: Parámetros de análisis establecidos en SciMAT.

\begin{tabular}{|c|c|}
\hline Configuración & Valores \\
\hline Unidad de análisis & $\begin{array}{c}\text { Palabras clave autores, palabras } \\
\text { clave Wos }\end{array}$ \\
\hline Umbral de frecuencia & P1 (2), P2 (2), P3 (2) \\
\hline Tipo de red & Co-ocurrencia \\
\hline $\begin{array}{c}\text { Umbral valor unión co- } \\
\text { ocurrencia }\end{array}$ & Índice de equivalencia (3), P3 (3) \\
\hline Medida de normalización & Tamaño máximo: 9 ;amaño \\
Algoritmo de clustering & mimo: 3 \\
\hline Medida evolutiva & Índice de Jaccard \\
\hline Medida superpuesta & Índinclusión \\
\hline
\end{tabular}




\section{Resultados}

Se ha llevado a cabo un análisis cuantitativo de una muestra $n=2978$ referencias (artículos, libros, capítulos de libro, proceedings, revisiones, etc.) siendo la mayoría de ellas provenientes de la búsqueda combinada LTU $(54,43 \%)$. Por otro lado, tal y como podemos observar en la siguiente tabla, el análisis de la producción diacrónica determina que es en la última década (2010-2019) donde tiene cabida la mayor producción científica al respecto $(87,2 \%)$, por lo que nos encontramos ante un tema en fase de crecimiento exponencial y de gran relevancia.

Tabla 3: Producción diacrónica (1900-2019)

\begin{tabular}{|l|c|c|c|c|c|c|c|}
\hline & LEU & LIU & LTU & LEHE & LIHE & LTHE & $\boldsymbol{\Sigma}$ \\
\hline $1900-1909$ & 1 & - & - & - & - & - & 1 \\
\hline $1910-1919$ & - & - & - & - & - & - & - \\
\hline $1920-1929$ & - & - & - & - & - & - & - \\
\hline $1930-1939$ & - & - & - & - & - & - & - \\
\hline $1940-1949$ & - & 1 & - & - & - & - & 1 \\
\hline $1950-1959$ & - & 1 & - & - & - & - & 1 \\
\hline $1960-1969$ & - & - & 3 & - & - & - & 3 \\
\hline $1970-1979$ & - & - & 3 & - & - & - & 3 \\
\hline $1980-1989$ & - & 4 & 2 & - & - & - & 6 \\
\hline $1990-1999$ & 6 & 11 & 23 & - & 3 & 3 & 46 \\
\hline $2000-2009$ & 59 & 39 & 170 & 8 & 9 & 34 & 319 \\
\hline $2010-2019$ & 441 & 116 & 1420 & 185 & 47 & 389 & 2598 \\
\hline Total & 507 & 172 & 1621 & 193 & 59 & 426 & 2978 \\
\hline
\end{tabular}

En cuanto a la productividad geográfica, un total de 21 países han superado los requisitos de inclusión, convirtiéndose así en los más prolíficos en cuanto a la investigación analizada (Tabla 4). Encontramos, de este modo, una mayor producción científica proveniente de Turquía $(12,2 \%)$, Estados Unidos $(9,4 \%)$, China $(9,2 \%)$ y Rusia $(7,7 \%)$.

Tabla 4: Países con mayor producción científica $(x \geq 20)$

\begin{tabular}{|l|c|c|c|c|c|c|c|}
\hline \multicolumn{1}{|c|}{ Cabecera } & LEU & LIU & LTU & LEHE & LIHE & LTHE & $\boldsymbol{\Sigma}$ \\
\hline Turquía & 60 & - & 248 & 20 & - & 35 & 363 \\
\hline Estados Unidos & 50 & 67 & 119 & - & 22 & 23 & 281 \\
\hline China & 38 & - & 161 & 24 & - & 50 & 273 \\
\hline Rusia & 44 & - & 143 & - & - & 42 & 229 \\
\hline España & - & - & 108 & - & - & 47 & 155 \\
\hline Inglaterra & - & - & 71 & - & - & 22 & 93 \\
\hline Japón & 43 & - & 43 & - & - & - & 86 \\
\hline Australia & 33 & - & 40 & - & - & - & 73 \\
\hline Irán & - & - & 65 & - & - & - & 65 \\
\hline República Checa & - & - & 57 & - & - & - & 57 \\
\hline Brasil & - & - & 44 & - & - & - & 44 \\
\hline Canadá & 20 & - & 23 & - & - & - & 43 \\
\hline Colombia & - & - & 33 & - & - & - & 33 \\
\hline
\end{tabular}




\begin{tabular}{|l|c|c|c|c|c|c|c|}
\hline Francia & - & - & 30 & - & - & - & 30 \\
\hline Rumanía & - & - & 30 & - & - & - & 30 \\
\hline Italia & - & - & 25 & - & - & - & 25 \\
\hline Malasia & - & - & 21 & - & - & - & 21 \\
\hline Polonia & - & - & 21 & - & - & - & 21 \\
\hline Mexico & - & - & 21 & - & - & - & 21 \\
\hline Taiwán & - & - & 19 & - & - & - & 19 \\
\hline
\end{tabular}

Por otro lado, en cuanto al análisis de las instituciones más prolíficas (Tabla 5), destaca la Kazan Federal University (Rusia) con un total de 61 referencias $(2,1 \%)$ como la organización con más producción científica al respecto.

Estos datos, a su vez, reflejan que el grueso del cuerpo científico se encuentra disperso entre distintas instituciones a nivel internacional. Finalmente, cabe señalar que las búsquedas LIU, LEHE, LIHE y LTHE no cumplen con los requisitos de inclusión.

Tabla 5: Instituciones con mayor producción científica $(x \geq 10)$.

\begin{tabular}{|l|c|c|c|c|}
\hline \multicolumn{1}{|c|}{ Institución } & País & LEU & LTU & $\boldsymbol{\Sigma}$ \\
\hline Kazan Federal University & Rusia & 21 & 40 & 61 \\
\hline Islamic Azad University & Irán & - & 29 & 29 \\
\hline Tomsk Polytechnic University & Rusia & - & 24 & 24 \\
\hline Hacettepe University & Turquía & - & 22 & 22 \\
\hline University of Hradec Kralove & República Checa & - & 22 & 22 \\
\hline Anadolu University & Turquía & - & 17 & 17 \\
\hline Gazi University & Turquía & - & 16 & 16 \\
\hline Sultan Qaboos University & Omán & - & 16 & 16 \\
\hline Open University UK & Inglaterra & - & 13 & 13 \\
\hline Tomsk State University & Rusia & - & 13 & 13 \\
\hline Cukurova University & Turquía & - & 11 & 11 \\
\hline
\end{tabular}

En cuanto a la productividad de autores, al análisis realizado para valorar a los expertos en la temática muestra que solamente tres autores (Tabla 6) provenientes de la búsqueda combinada LTU han superado los criterios de inclusión establecidos, convirtiéndose así en los más productivos sobre la enseñanza de lenguas en Web of Science.

Tabla 6: Autores más productivos $(x \geq 5)$.

\begin{tabular}{|l|c|c|c|}
\hline \multicolumn{1}{|c|}{ Cabecera } & Institución & País & LTU \\
\hline Hubackova, S. & University of Hradec Kralove & República Checa & 9 \\
\hline Galloway, N. & University of Edinburgh & Escocia & 5 \\
\hline Ozcelik, N. & Gazi University & Turquía & 5 \\
\hline Tuzlukova, V. & Sultan Qaboos University & Omán & 5 \\
\hline
\end{tabular}

En cuanto a la productividad de fuente, y más concretamente, respecto al tipo de producción, son los artículos, comunicaciones y/o ponencias en congresos y los capítulos de libro (Tabla 7 ) los más usuales al respecto. No obstante, es el primer grupo $(65,7 \%)$ el que conforma el grueso de la producción analizada sobre este campo. 
Tabla 7: Tipo de producción científica $(x \geq 20)$.

\begin{tabular}{|l|c|c|c|c|c|c|c|}
\hline & LEU & LIU & LTU & LEHE & LIHE & LTHE & $\boldsymbol{\Sigma}$ \\
\hline Artículo & 345 & 130 & 1060 & 127 & 38 & 256 & 1956 \\
\hline Proceedings & 153 & 38 & 554 & 60 & 20 & 163 & 988 \\
\hline Capítulo de libro & 21 & - & 32 & 15 & - & 14 & 82 \\
\hline
\end{tabular}

Por lo que corresponde al núcleo de la producción científica (Tabla 8), es decir, la fuente de los textos científicos que mayor suma de obras concentra, los análisis realizados determinan que la colección: Procedia Social and Behavioral Sciences (6,4\%), INTED Proceedings $(3,8 \%)$, ambas publicaciones resultantes de reuniones científicas, centralizan el mayor número de referencias $(10,2 \%)$. A su vez, destacan las revistas: Advances in Social Science Education and Humanities Research (1,5\%) y Modern Journal of Language Teaching Methods (1,3\%).

Tabla 8: Núcleo de la producción científica $(x \geq 15)$.

\begin{tabular}{|c|c|c|c|c|c|c|c|}
\hline Cabecera & LEU & LIU & LTU & LEHE & LIHE & LTHE & $\Sigma$ \\
\hline $\begin{array}{l}\text { Procedia Social } \\
\text { and Behavioral } \\
\text { Sciences }\end{array}$ & 21 & - & 147 & - & - & 22 & 190 \\
\hline $\begin{array}{l}\text { INTED } \\
\text { Proceedings }\end{array}$ & 25 & - & 64 & - & - & 22 & 111 \\
\hline $\begin{array}{l}\text { Advances in Social } \\
\text { Science Education } \\
\text { and Humanities } \\
\text { Research }\end{array}$ & - & - & 35 & - & - & 15 & 50 \\
\hline $\begin{array}{l}\text { Modern Journal of } \\
\text { Language } \\
\text { Teaching Methods }\end{array}$ & - & - & 44 & - & - & - & 44 \\
\hline $\begin{array}{l}\text { EDULEARN } \\
\text { Proceedings }\end{array}$ & - & - & 40 & - & - & - & 40 \\
\hline $\begin{array}{l}\text { Arab World English } \\
\text { Journal }\end{array}$ & - & - & 25 & - & - & - & 25 \\
\hline ICERI Proceedings & - & - & 22 & - & - & - & 22 \\
\hline $\begin{array}{l}\text { Journal of } \\
\text { Teaching English } \\
\text { for Specific and } \\
\text { Academic } \\
\text { Purposes }\end{array}$ & - & - & 19 & - & - & - & 19 \\
\hline $\begin{array}{l}\text { Foreign Language } \\
\text { Annals }\end{array}$ & - & - & 18 & - & - & - & 18 \\
\hline $\begin{array}{l}\text { Language } \\
\text { Learning in Higher } \\
\text { Education }\end{array}$ & - & - & 16 & - & - & - & 16 \\
\hline System & - & - & 16 & - & - & - & 16 \\
\hline $\begin{array}{l}\text { Yazyk I Kultura } \\
\text { Language and } \\
\text { Culture }\end{array}$ & - & - & 16 & - & - & - & 16 \\
\hline $\begin{array}{l}\text { International } \\
\text { Journal of English }\end{array}$ & - & - & 15 & - & - & - & 15 \\
\hline
\end{tabular}




\begin{tabular}{|l|l|l|l|l|l|l|l|}
\hline Linguistics & & & & & & & \\
\hline Porta Linguarum & - & - & 15 & - & - & - & 15 \\
\hline $\begin{array}{l}\text { Recherche et } \\
\text { pratiques } \\
\text { pedagogiques en } \\
\text { langues de } \\
\begin{array}{l}\text { specialite cahiers } \\
\text { de I apliut }\end{array}\end{array}$ & - & - & 15 & - & - & - & 15 \\
\hline
\end{tabular}

El grueso de la investigación científica se escribe y publica en inglés (86,33\%), tanto en la globalidad de los resultados, como en las seis búsquedas aisladas realizadas (Tabla 9). De este modo, el idioma de Shakespeare es el preferido para difundir el conocimiento sobre la enseñanza de lenguas.

Tabla 9: Idioma de publicación $(x \geq 20)$.

\begin{tabular}{|l|c|c|c|c|c|c|c|}
\hline Cabecera & LEU & LIU & LTU & LEHE & LIHE & LTHE & $\boldsymbol{\Sigma}$ \\
\hline Inglés & 450 & 152 & 1384 & 174 & 56 & 355 & 2571 \\
\hline Español & - & - & 70 & - & - & 26 & 96 \\
\hline Ruso & 23 & - & 40 & - & - & - & 59 \\
\hline Portugués & - & - & 36 & - & - & 13 & 49 \\
\hline Francés & - & - & 30 & - & - & - & 30 \\
\hline Turco & - & - & 24 & - & - & - & 24 \\
\hline
\end{tabular}

La investigación analizada proviene mayormente de tres áreas de investigación: educación, lingüística y otros tópicos relacionados con las Ciencias Sociales (Tabla 10). No obstante, la primera de ellas es la que concentra la mayor cuantía de referencias $(72,26 \%)$, tanto en la globalidad de los resultados como en las seis búsquedas combinadas.

Tabla 10: Área de investigación $(x \geq 10)$

\begin{tabular}{|l|c|c|c|c|c|c|c|}
\hline & LEU & LIU & LTU & LEHE & LIHE & LTHE & $\boldsymbol{\Sigma}$ \\
\hline $\begin{array}{l}\text { Education } \\
\text { \&ducationa }\end{array}$ & 328 & 112 & 1093 & 137 & 42 & 304 & 2152 \\
$\begin{array}{l}\text { I Research } \\
\text { Linguistics }\end{array}$ & 178 & 71 & 500 & 52 & 13 & 92 & 906 \\
\hline $\begin{array}{l}\text { Social } \\
\text { Sciences } \\
\text { Other } \\
\text { Topics }\end{array}$ & 27 & - & 142 & 14 & - & 40 & 223 \\
\hline
\end{tabular}

Respecto a las obras de referencia y que mayor impacto han suscitado entre la comunidad científica por su número de citas $(x \geq 100)$ los análisis pertinentes encuentran solamente tres obras que cumplan con los criterios de inclusión anteriormente mencionados. Así, de todas las búsquedas empleadas, el artículo de Blamey et al. (2001) (LTU) acerca de las relaciones entre la percepción del habla, la producción, el lenguaje, la pérdida de audición y la edad en niños con discapacidad auditiva es el que ha recibido 
mayor número de citas (229). La publicación de Hyland (2002) (LEU) es la segunda obra más citada (145) y cuyo objetivo se centra en la enseñanza del inglés para propósitos específicos (ESP). Finalmente, la investigación de Kelm (1992) (LIU) es la tercera más citada en la WOS (172) en este campo temático de investigación. Este aborda el uso de redes informáticas síncronas en la enseñanza de una segunda lengua.

Por otro lado, es preciso mencionar la investigación de Miyazone y Anderson (2010), pues, aunque no cumple con los requisitos de inclusión cabe destacar que es la investigación más reciente y que ha recibido mayor reconocimiento por parte de la comunidad científica (97 citas). El mismo trata sobre la enseñanza del inglés como lengua extranjera haciendo uso de entornos virtuales de comunicación y colaboración.

\subsection{Desarrollo estructural y temático}

La distribución de temas de los estudios sobre la enseñanza de idiomas en el ámbito universitario o la enseñanza superior en WoS se ha dividido en tres periodos, para analizar su avance durante toda su producción. La distribución de años se ha realizado tratando de mantener un número equitativos de documentos en los tres periodos.

En relación a las palabras clave y su evolución durante los tres periodos establecidos, se muestra que, durante el primer periodo, comprendido entre los años 1944 y 1999 , el número total de palabras clave es de 841 . Del primer al segundo periodo se observa que salen 537 palabras clave y se incluyen 2216 palabras nuevas. La coincidencia entre el primero periodo y el segundo en relación a estas palabras es del $36 \%$. En el segundo periodo el número de palabras clave es de 2520 . Del segundo al tercer periodo salen 1732 palabras clave y se introducen 2793 palabras nuevas, habiendo una coincidencia entre el segundo y el tercer periodo del 31\%. En el tercer periodo el número de palabras clave es de 12083, tal y como se muestra en la Figura 1.

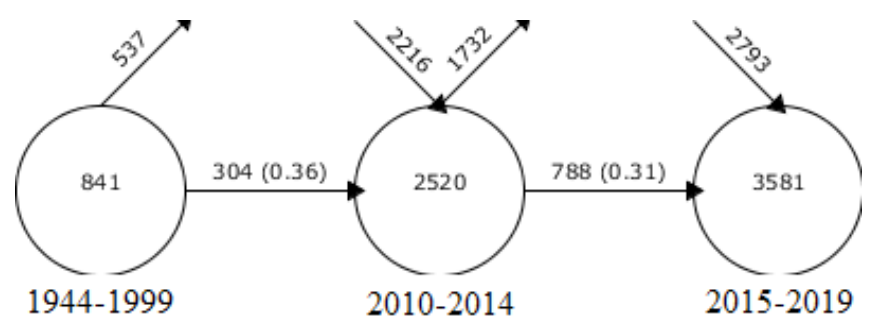

Figura 1: Continuidad de palabras clave entre periodos contiguos.

Iniciando el análisis de los temas de investigación en los distintos periodos, se observa que en el primero, de 1944 hasta 1999, los temas con mayor índice son TECHNOLOGY, FOREIGN LANGUAGE y SPANISH (tabla 11).

Tabla 11: Rendimiento temático en el periodo 1944-2009.

\begin{tabular}{|l|c|c|c|c|c|c|}
\hline Nombre & Obras & Índice-h & Índice-g & Índice-hg & Índice-q2 & Citas \\
\hline Technology & 4 & 4 & 4 & 4 & 9.59 & 88 \\
\hline On-line courses & 2 & 2 & 2 & 2 & 7.62 & 58 \\
\hline Foreign & 5 & 4 & 4 & 4 & 6 & 74 \\
\hline
\end{tabular}




\begin{tabular}{|l|c|c|c|c|c|c|}
\hline language & & & & & & \\
\hline $\begin{array}{l}\text { Higher } \\
\text { education }\end{array}$ & 5 & 2 & 3 & 2.45 & 4.47 & 17 \\
\hline University & 3 & 1 & 1 & 1 & 4.9 & 24 \\
\hline Spanish & 4 & 4 & 4 & 4 & 6.93 & 49 \\
\hline Attitudes & 3 & 2 & 3 & 2.45 & 6.78 & 39 \\
\hline
\end{tabular}

En este mismo periodo, los temas motores son SPANISH y TECHNOLOGY. Al examinar cada una de estos temas, se comprueba que los estudios sobre SPANISH se centran en COMPREHENSION y NATIVE SPEAKERS. En cambio, TECHNOLOGY se fundamenta en TEACHER TRAINING, LANGUAGE INSTRUCTION, LANGUAGE, CLASSROOM Y BARRIERS. En este periodo se muestra que el tema HIGHER EDUCATION es una incógnita, dado que su ubicación lo posiciona como un tema emergente o que tiende a desaparecer (Figura 2).

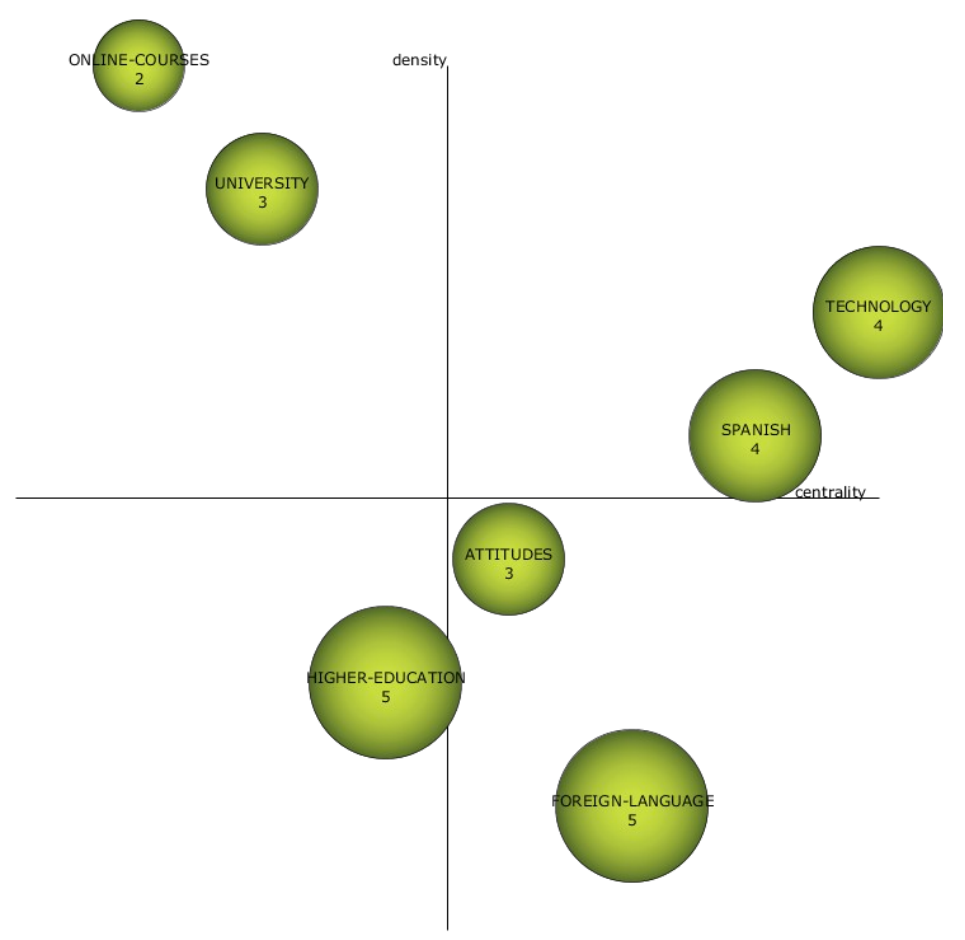

Figura 2: Diagrama estratégico por citas para el periodo 1944-1999.

En el segundo periodo, comprendido entre los años 2010 y 2014, ambos inclusive, el tema con mayor índice es LANGUAGE (Tabla 12).

Tabla 12: Rendimiento temático en el periodo 2010-2014

\begin{tabular}{|l|c|c|c|c|c|c|}
\hline Nombre & Obras & Índice-h & Índice-g & Índice-hg & Índice-q2 & Citas \\
\hline Language & 32 & 8 & 15 & 10.95 & 14.14 & 238 \\
\hline E-learning & 22 & 3 & 5 & 3.87 & 4.24 & 32 \\
\hline Technology & 10 & 3 & 3 & 3 & 3.87 & 16 \\
\hline Motivation & 14 & 5 & 7 & 5.92 & 6.32 & 62 \\
\hline
\end{tabular}




\begin{tabular}{|l|c|c|c|c|c|c|}
\hline $\begin{array}{l}\text { 2nd } \\
\text { language }\end{array}$ & 7 & 5 & 6 & 5.48 & 5.48 & 40 \\
\hline $\begin{array}{l}\text { Foreing } \\
\text { language }\end{array}$ & 6 & 1 & 1 & 1 & 1 & 2 \\
\hline $\begin{array}{l}\text { Language } \\
\text { teaching }\end{array}$ & 6 & 2 & 3 & 2.45 & 3.16 & 10 \\
\hline
\end{tabular}

Analizando el diagrama de la Figura 3, se muestran tres temas motores en este periodo: 2ND LANGUAGE, E-LEARNING y LANGUAGE. Las investigaciones sobre 2ND LANGUAGE se centran en L2, WILLIGNES y LEARNERS. Las de E-LEARNING se basan en ESP, ELT, STUDENTS GROUP, ICT, LEARNING STYLES, FOREIGN LANGUAGE TEACHING, ENGLISH LANGUAGE TEACHING y BLENDED LEARNING. Y las de LANGUAGE se centra en ACQUISITION, CULTURE, TEACHING, STUDENTS, UNIVERSITY, COMMUNICATION, ENGLISH y HIGHER EDUCATION. En este periodo se muestran dos temáticas, FOREIGN LANGUAGE y LANGUAGE TEACHING, consideradas incógnitas, dada su posición en el diagrama, no pudiendo determinar si son temas emergentes o tienden a desaparecer.

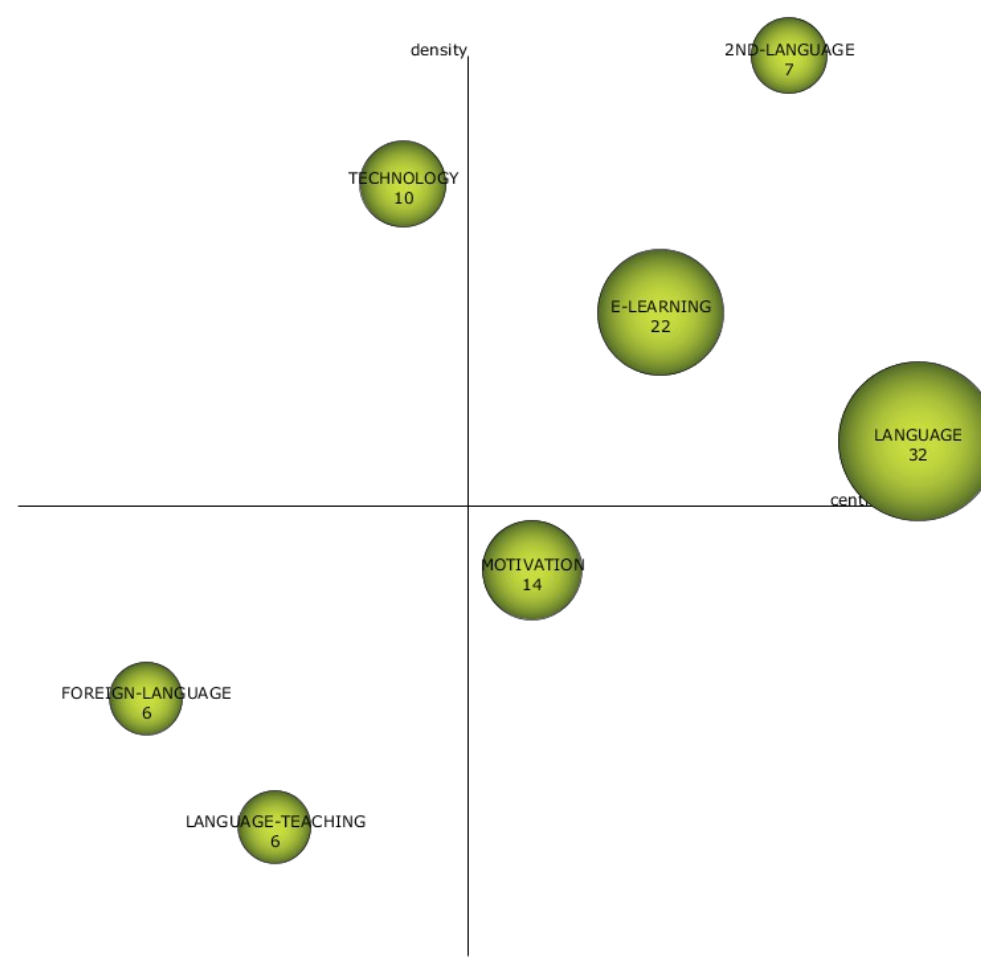

Figura 3: Diagrama estratégico por citas para el periodo 2000-2014.

En el último y tercer periodo, comprendido entre los años 2015 y 2019, los temas con mayor índice son STUDENTS, HIGHER EDUCATION y LANGUAGE (Tabla 13). 
Tabla 13: Rendimiento temático en el periodo 2015-2019.

\begin{tabular}{|l|c|c|c|c|c|c|}
\hline Nombre & Obras & Índice-h & Índice-g & Índice-hg & Índice-q2 & Citas \\
\hline Students & 81 & 6 & 8 & 6.93 & 8.12 & 118 \\
\hline $\begin{array}{l}\text { Higher } \\
\text { education }\end{array}$ & 41 & 5 & 7 & 5.92 & 6.71 & 66 \\
\hline Language & 20 & 4 & 7 & 5.29 & 6.93 & 59 \\
\hline Technology & 24 & 3 & 4 & 3.46 & 3.46 & 25 \\
\hline Beliefs & 27 & 3 & 4 & 3.46 & 4.24 & 26 \\
\hline Instruction & 16 & 2 & 3 & 2.45 & 3.16 & 15 \\
\hline $\begin{array}{l}\text { University } \\
\text { Education }\end{array}$ & 8 & 2 & 3 & 2.45 & 4.47 & 15 \\
\hline Fluency & 8 & 1 & 1 & 1 & 4 & 16 \\
\hline Policy & 8 & 1 & 2 & 1.41 & 2.24 & 6 \\
\hline Clil & 8 & 1 & 1 & 1 & 1 & 1 \\
\hline
\end{tabular}

Siguiendo lo marcado en el diagrama de la Figura 4, los temas motores son STUDENTS y HIGHER EDUCATION, aunque el tema BELIEFS se encuentra entre tema motor y tema básico y transversal. El tema STUDENTS centra sus investigaciones en TEACHING, LEARNING, SUCCES, TEACHERS, ATTITUDES, PERCEPTIONS, ENGLISH y EDUCATION. En cambio, el tema HIGHER EDUCATION se fundamenta en FOREIGN LANGUAGE EDUCATION, ENGLISH MEDIUM INSTRUCTIONS, WEB 2.0, LEARNERS, INTERNATIONALIZATIONS, LANGUAGE LEARNING, GLOBALIZATION y JAPAN. Además, durante este periodo hay un tema, CLIL, considerado como tema incógnita, dado que puede ser un tema emergente o que tiende a desaparecer.

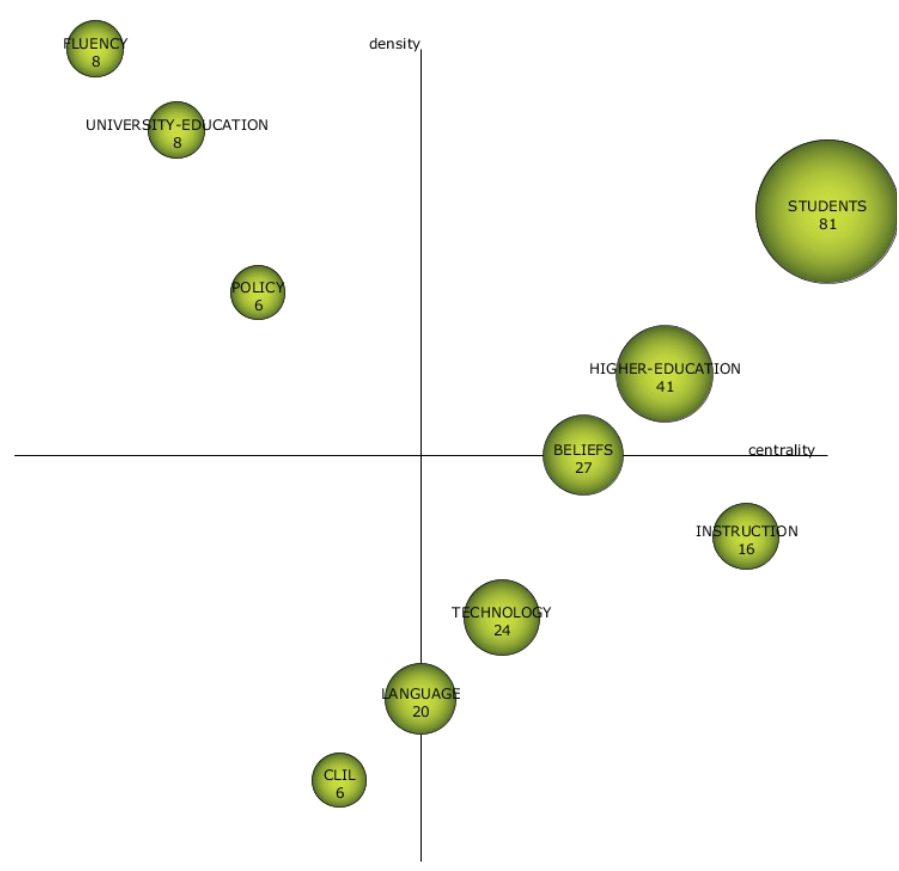

Figura 4: Diagrama estratégico por citas para el periodo 2015-2019. 


\subsection{Evolución de los temas}

La evaluación de los temas del estudio sobre la enseñanza de idiomas en el ámbito universitario, tal y como se muestra en la Figura 5, solamente muestra continuidad en TECHNOLOGY, habiendo brechas conceptuales en temas como FOREIGN LANGUAGE y HIGHER EDUCATION, dado que aparecen en dos de los tres periodos establecidos. Las líneas sólidas muestran que las relaciones son por temas, mientras que las líneas discontinuas ofrecen relaciones únicamente por palabras clave. A mayor tamaño del tema, mayor es el índice-h posee.

En el primer periodo, los temas con mayor índice-h son TECHNOLOGY, FOREIGN LANGUAGE y SPANISH. Las relaciones conceptuales que se establecen entre el primero y segundo periodo son TECHNOLOGY-LANGUAGE, TECHNOLOGY-TECHNOLOGY, FOREIGN LANGUAGE-FOREIGN LANGUAGE, UNIVERSITY-LANGUAGE, HIGHER EDUCATION-LANGUAGE y ATTITUDES-MOTIVATION. Las relaciones con mayor fuerza de unión son FOREIGN LANGUAGE-FEREIGN LANGUAGE y ATTITUDESMOTIVATION.

En el segundo periodo, el tema con mayor índice-h es LANGUAGE. Las relaciones conceptuales con el tercer periodo son LANGUAGE-STUDENTS, LANGUAGE-HIGHER EDUCATION, LANGUAGE-LANGUAGE, TECHNOLOGY-TECHNOLOGY, MOTIVATIONBELIEFS, FOREIGN LANGUAGE-BELIEFS y LANGUAGE TEACHING-TECHNOLOGY. La conexión con más fuerza es LANGUAGE TEACHING-TECHNOLOGY.

En el tercer periodo el tema con mayor índice-h es STUDENTS. En este caso, las relaciones conceptuales con el anterior periodo son STUDENTS-LANGUAGE, HIGHER EDUCATION-LANGUAGE, LANGUAGE-LANGUAGE, TECHNOLOGY-TECHNOLOGY, TECHNOLOGY-LANGUAGE TEACHING, BELIEFS-MOTIVATION.

En los tres periodos aparecen temas que no tienen conexión alguna, además aparecer de forma esporádica en uno de ellos. 


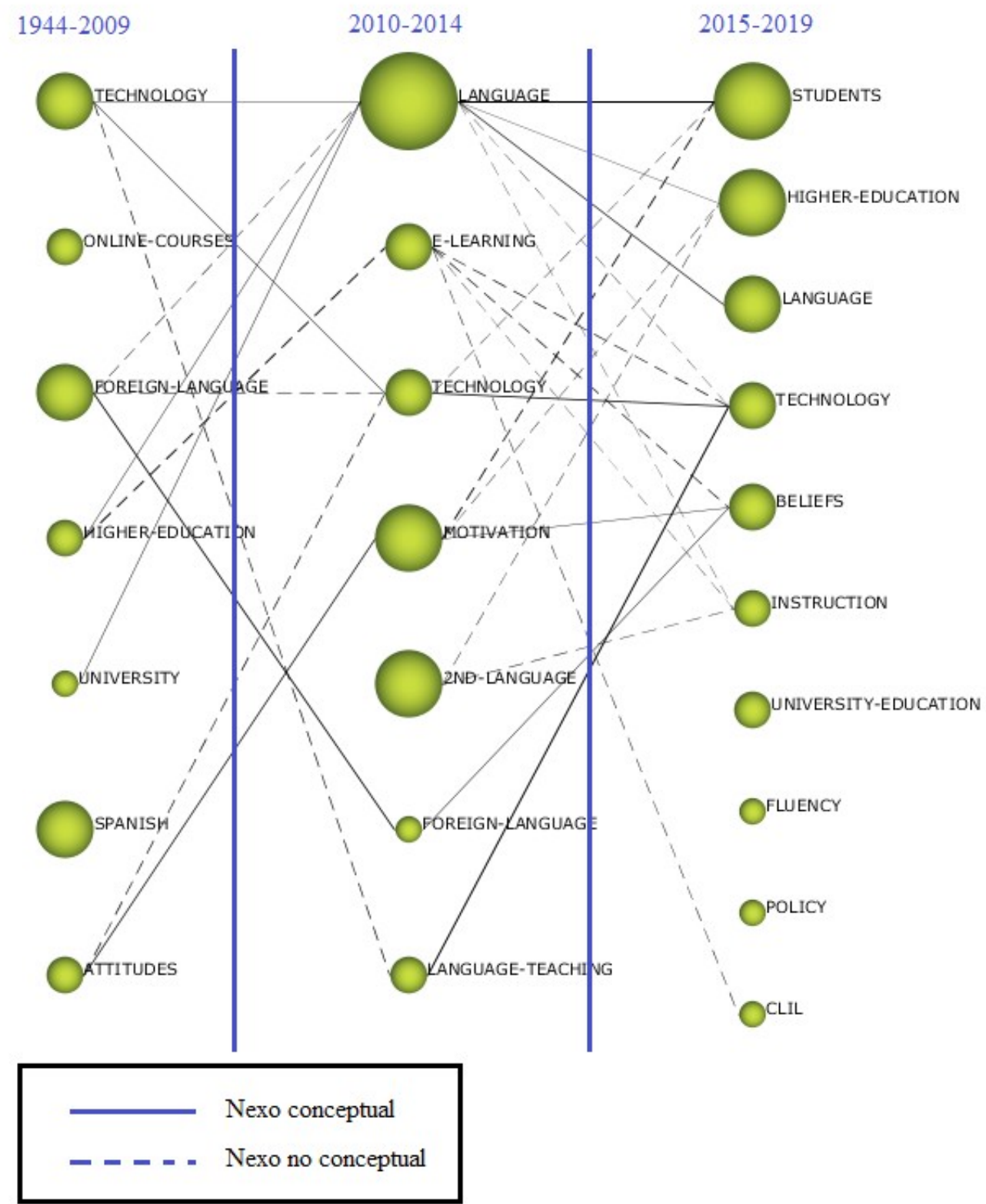

Figura 5: Evolución de temas por índice-h.

\section{Discusión y conclusiones}

Los análisis realizados determinan un patrón de crecimiento exponencial de la literatura científica sobre la enseñanza de idiomas en el ámbito universitario durante la última década (2010-2019). Nos encontramos, pues, ante un tema muy relevante y de gran actualidad que suscita interés a nivel internacional.

Se ha hallado una mayor producción científica en la colección principal de la Web of Science que proviene de investigadores de Turquía, siendo este el país que más ha producido al respecto. Destacan, a su vez, estudios de Estados Unidos, China, Rusia y España. A su vez, cuatro universidades de Turquía (Anadolu University; Cukurova 
University; Gazi University; Hacettepe University) se encuentran dentro de las instituciones más productivas científicamente. Sin embargo, por su mayor cuantía de trabajos publicados al respecto, destacamos la labor de la universidad rusa Kazan Federal University.

Respecto a la autoría de los trabajos, no se han encontrado autores verdaderamente prolíficos al respecto, salvo la autora checa Sarka Hubackova, de la University of Hradec Kralove, que cuenta con un total de nueve publicaciones al respecto.

Por otro lado, se mantiene una tendencia a publicar mayoritariamente a través de artículos y en inglés sobre algún ámbito de la investigación educativa, algo que no resulta de extrañar si tenemos en cuenta los criterios de promoción para investigadores en la actualidad, el idioma científico ampliamente aceptado y el contexto del tema de investigación.

Existe evolución de temas en el estudio de la enseñanza de idiomas y la educación superior, centrada en TECHNOLOGY únicamente, también se visualiza brechas conceptuales en temas como LANGUAGE, HIGHER EDUCATION y LANGUAGE. La evolución de temas muestra conexiones conceptuales consolidadas en el tiempo, tanto de los mismo temas como en otros.

Después de analizar las temáticas dadas después de aplicar el mapeo conceptual sobre la temática tratada, se puede concluir:

- TECHNOLOGY: Es el único tema que está presente en los tres periodos, siendo tema motor en el primer periodo, tema muy desarrollado y aislado en el segundo, y tema básico y transversal en el tercero. En el primer periodo es uno de los que presenta el índice-h más elevado. La relación establecida con el segundo periodo es conceptual con LANGUAGE y TECHNOLOGY, entre el segundo y primer periodo es conceptual con TECHNOLOGY, entre el segundo y tercer periodo es conceptual con TECHNOLOGY, y entre el tercer y segundo periodo es conceptual con TECHNONOLOGY y LANGUAGE TEACHING. Es un tema en decadencia.

- ONLINE-CURSES: Aparece únicamente en el primer periodo, como un tema muy desarrollado y aislado. No muestra conexión de ningún tipo con el segundo periodo. Es un tema en decadencia.

- FOREIGN-LANGUAGE: Este tema aparece en el primer periodo, como tema básico y transversal, y en el segundo periodo como tema emergente 0 en desaparición. En el primer periodo es uno de los temas con mayor índice-h. Su conexión con entre el primer y segundo periodo es conceptual con FOREIGN LANGUAGE, entre el segundo y primer periodo es conceptual con FOREIGN LANGUAGE, y entre el segundo y tercer periodo es conceptual con BELIEFS. Es un tema en decadencia.

- HIGHER EDUCATION: Aparece en el primer periodo, como tema emergente o en desaparición, en el tercer periodo, como tema motor. Este es un claro ejemplo de la brecha conceptual existente en este tema durante su evolución en el tiempo analizado. La conexión entre el primer y segundo periodo es conceptual con LANGUAGE, entre el tercer y segundo periodo es conceptual con LANGUAGE. Es un tema en auge y de gran relevancia para la comunidad científica.

- UNIVERSITY: Aparece solamente en el primer periodo, como un tema muy desarrollado y aislado. La conexión entre el primer y segundo periodo es conceptual con LANGUAGE. Es un tema en decadencia. 
- SPANISH: Este tema aparece en el primero periodo únicamente, siendo tema motor, además de ser uno de los que mayor índice-h presenta. No tiene conexión de ningún tipo con el segundo periodo. Es un tema en decadencia.

- ATTITUDES: Surge en el primer periodo exclusivamente. Su conexión con el segundo periodo es conceptual con MOTIVATION. Es un tema en decadencia.

- LANGUAGE: Este tema aparece en el segundo periodo, como tema motor, y en el tercer periodo, como tema situado entre emergente o en desaparición, y básico y transversal. En el segundo periodo es el tema con mayor índice-h. La conexión establecida entre el segundo y primer periodo es conceptual con TECHNOLOGY, HIGHER EDUCATION y UNIVERSITY, entre el segundo y tercer periodo es conceptual con STUDENTS, HIGHER EDUCATION y LANGUAGE y entre el tercer y el segundo periodo es conceptual con LANGUAGE. Es un tema en decadencia.

- E-LEARNING: Aparece solamente en el segundo periodo como tema motor. La conexión entre el segundo y primer periodo es no conceptual con HIGHER EDUCATION, y entre el segundo y tercer periodo es no conceptual con TECHNOLOGY, BELIEFS, INSTRUCTION y CLIL. Es un tema en decadencia.

- MOTIVATION: Surge en el segundo periodo como un tema básico y transversal. La conexión entre el segundo y primer periodo es conceptual con ATTITUDES y entre el segundo y tercer periodo es conceptual con BELIEFS. Es un tema en decadencia.

- $2^{\circ}$ ND LANGUAGE: Este tema aparece únicamente en el segundo periodo como tema motor. No mantiene conexión alguna con el primer periodo. En cambio, con el tercer periodo establece una relación no conceptual con HIGHER EDUCATION e INSTRUCTION. Es un tema en decadencia.

- LANGUAGE TEACHING: Aparece en el segundo periodo como un tema emergente o en desaparición. Tanto con el primer como en el tercer periodo, la conexión es con el tema TECHNOLOGY, aunque con el primer periodo es no conceptual y con el tercero es conceptual. Es un tema en decadencia.

- STUDENTS: Surge únicamente en el tercer periodo, como tema motor y con mayor índice-h. Su conexión con el segundo periodo es conceptual con LANGUAGE. Es un tema en auge y de gran relevancia en las investigaciones actualmente.

- BELIEFS: Aparece en el tercer periodo como tema situado entre motor, y básico y transversal. La conexión con el segundo periodo es conceptual con MOTIVATION y FOREIGN LANGUAGE. Es un tema de interés y relevancia para la comunidad científica.

- INSTRUCTION: Surge en el tercer periodo como tema básico y transversal. La conexión con el segundo periodo es no conceptual con LANGUAGE, E-LEARNING y 2ND LANGUAGE. Es un tema en decadencia.

- UNIVERSITY EDUCATION: Aparece en el tercer periodo como un tema muy desarrollado y aislado. No muestra conexión alguna con el segundo periodo. Es un tema en decadencia.

- FLUENCY: Surge en el tercer periodo como un tema muy desarrollado y aislado. No presenta conexión alguna con el segundo periodo. Es un tema en decadencia.

- POLICY: Solamente aparece en el tercer periodo como un tema muy desarrollado y 
aislado. No presenta conexión alguna con el segundo periodo. Es un tema en decadencia.

- CLIL: Aparece en el tercer periodo como un tema emergente o en desaparición. La conexión establecida con el segundo periodo es no conceptual con E-LEARNING.

Es un tema incógnita en los próximos años, dado que no puede emerger o desaparecer.

Se puede finalizar indicando que las investigaciones sobre la enseñanza de idiomas en el ámbito universitario están empezando a centrarse en la educación superior, en los propios estudiantes y en las creencias que los docentes puedan tener sobre el alumnado. Los temas HIGHER EDUCATION, tal y como reflejan Durham y Kim (2019), STUDENTS, como los estudios realizados por Hafner (2015) y Beliefs, orientados a investigaciones como las de Risueño et al. (2016), pueden ser consideradas las bases de investigación de los próximos años dentro de este campo de estudio.

Las limitaciones encontradas en el desarrollo de esta investigación ha sido la depuración de la base de datos y la organización de los temas de investigación, dada la gran cantidad de palabras clave que acumulan toda las referencias analizadas y las incorrecciones ortográficas encontradas en muchas de ellas.

Como futura línea de investigación, se puede desarrollar el mismo análisis en otras bases de datos, tales como Google Academy o Scopus, dado que la base de datos WoS se centra principalmente en investigaciones desarrolladas en el ámbito anglosajón.

\section{Referencias}

ÁLVAREZ, G. La educación comparada más allá de la REEC: análisis bibliométrico de la disciplina en las cinco revistas españolas afines de más impacto entre 1995 y 2014 . Revista Española de Educación Comparada, n. 25, p. 19-45, 2015 . doi: 10.5944/reec.25.2015.14782

CALLON, M.; COURTIAL, J. P.; LAVILLE, F. Co-word analysis as a tool for describing the network of interactions between basic and technological research: The case of polumer chemsitry. Sciencitometric, v. 1, n. 22, p. 155-205, 1991. doi: 10.1007/BF02019280

COBO, M. J. LÓPEZ, A. G.; HERRERA, E.; HERRERA, F. Science mapping software tools: Review, analysis, and cooperative study among tools. Journal of the American Society for Information Science and Technology, v. 62, n.7, p. 1382-1402, 2011. doi: 10.1002/asi.21525

COBO, M. J.; LÓPEZ, A. G.; HERRERA, E.; HERRERA, F. SciMAT: A new science mapping analysis software tool. Journal of American Society for Information Science and Technology, v.63, n. 8, p. 1609-1630, 2012. doi: 10.1002/asi.22688

DURHAM, L.; KIM, S. Training dilemmas and recommendations with volunteer instructors in small, faith-based adult ESL programs. TESOL Journal, v. 10, n.1, p. 1-11, 2019.

CROMPTON, H.; BURKE, D. The use of mobile learning in higher education: A systematic review. Computers \& Education, n. 123, p. 53-64, 2018. 
ELKINS, M. R.; MAHER, C. G.; HERBERT, R. D. MOSELEY, A. M.; SHERRINGTON, C. Correlation between the journal impact factor and three other journal citation indices. Scientometrics, n. 85, p. 81-93, 2010. doi: 10.1007/s11192-010-0262-0

FRANCESCHET, M. Journal influence factors. Journal of Informetrics, v. 3, n. 4, p. 239-248, 2010. doi: 10.1016/j.joi.2009.12.002

FRANCIOSI, S. The Effect of Computer Game-Based Learning on FL Vocabulary Transferability. Educational Technology \& Society, v. 20, n. 1, p. 123-133, 2017.

GIMÉNEZ-ESPERT, M. C.; PRADO-GASCÓ, V. J. Bibliometric analysis of six nursing journals from the Web of Science, 2012-2017. Journal of advanced nursing, 75(3), p. 543554, 2019.

GROVES. M.; MUNDT, K. Friend or foe? Google Translate in language for academic purposes. English for specific purporses, n. 37, p. 112-121, 2015 doi: 10.1016/j.esp.2014.09.001

HAFNER, C. A. Remix Culture and English Language Teaching: The Expression of Learner Voice in Digital Multimodal Compositions. Tesol quarterly, v. 49, n.3, p. 486-509, 2015. doi: 10.1002/tesq.238

HINOJO, F. J.; AZNAR, I.; ROMERO, J. M. Dispositivos móviles para el aprendizaje: análisis de la investigación doctoral sobre mobile learning en España. Texto livre: Linguagem e Tecnologia, v. 11, n. 3, p. 154-175, 2018. http://dx.doi.org/10.17851/1983$\underline{3652.11 .3 .154-175}$

HIRSCH, J. E. An index to quantify an individual's scientific research output. Proceedings of National Academy of Sciences, v. 102, n. 46, p. 16569-16572, 2005. doi: 10.1073/pnas.0507655102

HUNG, H. T.; YANG, J. C.; HWANG, G. J.; CHU, H. C.; WANG, C. C. A scoping review of research on digital game-based language learning. Computers \& Education, n. 126, p. 89104, 2018. doi: 10.1016/j.compedu.2018.07.001

HUTTO, B.; CATALÁ, F.; MOHER, D. La extensión de la declaración PRISMA para revisiones sistemáticas que incorporan metaanálisis en red: PRISMA-NMA. Medicina Clínica, v. 147, n. 6, p. 262-266, 2016. doi: 10.1016/j.medcli.2016.02.025

KING, J. "It's Time, Put on the Smile, It's Time!": The Emotional Labour of Second Language Teaching Within a Japanese University. En: GKONOU, C.; TATZL, D.; MERCER, S. (ed.), New directions in language learning phychology, p. 97-112, 2016. New York: Springer.

LANVERS, U. Contradictory Others and the Habitus of Languages: Surveying the L2 Motivation Landscape in the United Kingdom. Modern Language Journal, v. 101, n. 3, p. 517-532, 2017 doi: 10.1111/modl.12410 
LO BIANCO, J. Domesticating the foreign: globalization's effects on the place/s of languages. Modern Language Journal, n. 98, p. 312-325, 2014. doi: 10.1111/j.15404781.2014.12063.x

LOPEZ, E.; VAZQUEZ, E.; SARASOLA, J. L. Estudio Bibliométrico Pixel-Bit. Revista de Medios y Educación (2000-2013). Pixel-Bit. Revista de Medios y Educación, n. 46, p. 65-85, 2015. doi: 10.12795/pixelbit.2015.i46.05

MANTOU, N.; NOELS, K. A. Measuring Language Mindsets and Modeling Their Relations With Goal Orientations and Emotional and Behavioral Responses in Failure Situations. The Modern Language Journal, v. 101, n. 1, p. 212-243, 2017. doi: 10.1111/modl.12380.

MAHAPATRA, S.; MISHRA, S. Articulating identities - the role of English language education in Indian universities. Teaching in higher education, v. 24, n.3, 346-360, 2019. doi: 10.1080/13562517.2018.1547277

MONTERO, J.; COBO, M. J.; GUTIÉRREZ, M.; SEGADO, F.; HERRERA, E. Mapeo científico de la Categoría "Comunicación" en WoS (1980-2013). Comunicar, v. 55, n. 26, 8191, 2018. doi: 10.3916/C55-2018-08

QUEVEDO, R.; LÓPEZ, W. Análisis bibliométrico de las revistas multidisciplinares de psicología incorporadas en la Web of Science (2008-2009). Psicología: Reflexao e Crítica, v. 23, n. 2 , p. 384-408, 2010.

RODRÍGUEZ, A. M.; TRUJILLO, J. M.; SÁNCHEZ, J. Impacto de la productividad científica sobre competencia digital de los futuros docentes: aproximación bibliométrica en Scopus y Web of Science. Revista Complutense de Educación, v. 30, n. 2, p. 623-646, 2019. doi: 10.5209/RCED.58862

RODRÍGUEZ, A. M.; RASO F.; RUIZ, J. Competencia digital, educación superior y formación del profesorado: un estudio de meta-análisis en la Web of Science. Pixel-Bit. Revista de Medios de Comunicación, n. 54, p. 65-81, 2019. doi: 10.12795/pixelbit.2019.154.04

ROSE, H.; MCKINLEY, J. Japan's English-medium instruction initiatives and the globalization of higher education. Higher Education, v. 75, n.1, p. 111-129, 2018. doi: 10.1007/s10734-017-0125-1

RISUEÑO, J. J.; VÁZQUEZ, M. L.; HIDALGO, J.; DE LA BLANCA, S. Language learning strategy use by spanish efl students: the effect of proficiency level, gender, and motivation. Revista de Investigación Educativa, v. 34, n. 1, p. 133-149, 2016. doi: 10.6018/rie.34.1.232981.

RUIZ, R.; DELGADO, E.; JIMÉNEZ, E. Principios y criterios utilizados en España por la Comisión Nacional Evaluadora de la Actividad Investigadora (CNEAI) para la valoración de las publicaciones científicas: 1989-2009. Psicothema, n. 222, p. 898-908, 2010. 
TODESCHINI, R.; BACCINI, A. Handbook of Bibliometric Indicators: Quantitative Tools for Studying and Evaluating Research. Milano: Wiley-VCH, 2016.

VELASCO, B.; EIROS, J. M.; PINILLA, J. M.; SAN ROMÁN, J. A. La utilización de indicadores bibliométricos para evaluar la actividad investigadora. Aula Abierta, v. 40, n. 2, p. $75-84,2012$.

ZHENG, Y.; GUO, X. Publishing in and about English: challenges and opportunities of Chinese multilingual scholars' language practices in academic publishing. Language Policy, v. 18, n. 1, p. 107-130, 2019. doi: 10.1007/s10993-018-9464-8.

ZYCH, I.; BUELA, G. Internacionalidad de las revistas de Psicología Multidisciplinar editadas en Iberoamérica e incluidas en la Web of Science. Universitas Psychologica, n. 9, p. 27-34, 2010. Aprovado em dia 06 de outubro de 2019. 